\title{
The interaction with $\mathrm{H}-2 \mathrm{D}^{\mathrm{d}}$ in cis is associated with a conformational change in the Ly49A NK cell receptor
}

\author{
Jonathan Back ${ }^{1+}$, Georgi S. Angelov ${ }^{1}$, Roy A. Mariuzza ${ }^{2}$ and Werner Held ${ }^{1}$ * \\ ${ }^{1}$ Ludwig Center for Cancer Research of the University of Lausanne, Epalinges, Switzerland \\ ${ }^{2}$ WM Keck Laboratory for Structural Biology, University of Maryland Institute for Bioscience and Biotechnology Research, Rockville, MD, USA
}

\section{Edited by:}

Marco Colonna, Washington

University School of Medicine, USA

Reviewed by:

Laurel L. Lenz, National Jewish Health, USA

Petter Brodin, Karolinska Institute,

Sweden

\section{${ }^{*}$ Correspondence:}

Werner Held, Ludwig Center for Cancer Research of the University of Lausanne, Ch. des Boveresses 155,

1066 Epalinges, Switzerland.

e-mail:werner.Held@unil.ch

\section{${ }^{\dagger}$ Present address:}

Jonathan Back, Glenmark

Pharmaceuticals S.A., Chemin de la Combeta 5, 2300 La Chaux-de-Fonds, Switzerland.
Mouse natural killer (NK) cells express Ly49 family receptors that recognize major histocompatibility complex class I (MHC-I) molecules. By interacting with MHC-I molecules expressed on other cells (in trans), inhibitory Ly49 receptors prevent the NK cell-mediated killing of normal cells. In addition, some Ly49 receptors have the unusual property to also interact with MHC-I molecules expressed by the NK cell itself (in cis). cis Binding sequesters a significant fraction of the NK cells' Ly49 receptors, reducing the number of receptors available for trans binding. This lowers the threshold at which NK cell activation exceeds inhibition rendering NK cells more sensitive. It is unclear how Ly49 receptors can bind MHC-I in trans and in cis using the same binding site. We have proposed that this is mediated by two distinct conformations of Ly49 receptors. Here we have tested this model by inferring the distance between the ligand-binding domain of Ly $49 \mathrm{~A}$ and the cell membrane using fluorescence resonance energy transfer (FRET). Consistent with the concept, reducing the distance between the ligand-binding domain of Ly49A and the cell membrane, by shortening the Ly49A stalk, resulted in a substantially increased FRET. The co-expression of cognate MHC-I ligand reduced FRET derived from Ly49A variants with a shortened stalk, indicating that cis association alters FRET. Indeed, FRET improved when cis complexes were disrupted using acid-mediated destruction of $\mathrm{MHC}$-I complexes. These data provide direct evidence that the interaction with $\mathrm{MHC}-\mathrm{I}$ in cis is associated with a conformational change in the Ly49A receptor on the surface of live cells. The novel FRET based approach may be generally applicable to study conformational changes in cell surface receptors.

\section{Keywords: Ly49, MHC-I, conformation, FRET}

\section{INTRODUCTION}

Natural killer (NK) cells can be activated by multiple cell surface receptors that recognize ligands expressed by normal and diseased cells. Activation signaling is interrupted when inhibitory NK cell receptors engage major histocompatibility complex class I (MHCI) molecules on other cells. This dual receptor system enables NK cells to detect virally infected or transformed cells with reduced levels of MHC-I molecules. In this case, NK cell activation can proceed and induce the lysis of such "missing-self" cells.

Inhibitory NK cell receptors specific for MHC-I molecules include human leukocyte immunoglobulin (Ig)-like receptors (LILRs), killer Ig-like receptors (KIRs; human), C-type lectin-like Ly49 receptors (mouse), and CD94-NKG2A receptors (human and mouse; Lanier, 2005). Engagement of these receptors by MHC-I counteracts activation signaling in mature NK cells. In addition, in the presence of the cognate MHC-I ligand, KIR, and Ly49 receptors "educate" developing NK cells, i.e., they establish and/or maintain functional competence of activating NK cell receptors (Fernandez et al., 2005; Kim et al., 2005; Anfossi et al., 2006; Chalifour et al., 2009; Sola et al., 2009). The precise role of MHC-I receptors in NK cell education is still debated.

Ly49A represents the prototype MHC-I receptor on mouse NK cells. This receptor binds to $\mathrm{H}-2 \mathrm{D}^{\mathrm{d}}$ but not to $\mathrm{H}-2^{\mathrm{b}} \mathrm{MHC}$ class I molecules (Karlhofer et al., 1994). In addition to the classical binding of ligand expressed on other cells (in trans), Ly49A can also bind ligand expressed in the plane of the NK cell membrane (in cis; Doucey et al., 2004). Besides Ly49A, several other Ly49 family receptors can also bind MHC-I ligand expressed in cis (Scarpellino et al., 2007). Moreover, the structurally distinct, Ig-like, inhibitory human LILRB2 and the homologous mouse PIR-B receptors can also interact with MHC-I ligands expressed in trans as well as in cis (Masuda et al., 2007; reviewed in Held and Mariuzza, 2008). cis Binding of Ly49A reduces (by a factor of 3-4) the number of receptors that can functionally interact with $\mathrm{D}^{\mathrm{d}}$ expressed on potential target cells (Back et al., 2007). There is no evidence that cis binding of Ly49A results in inhibitory signaling (Doucey et al., 2004). Hence, cis binding lowers the threshold at which NK cell activation exceeds inhibition. This renders Ly49A NK cells more sensitive to react to diseased host cells. Further data suggest that cis interaction of Ly49A with MHC-I plays an essential role in NK cell education (Chalifour et al., 2009; Jonsson et al., 2010).

trans and cis Binding by Ly49 receptors is mediated by the same binding site (Doucey et al., 2004), suggesting that the ligandbinding natural killer domain (NKD) domain of Ly49 has to reverse its orientation relative to the NK cell membrane in order to bind MHC-I in cis versus trans. Indeed, a combination of 
structural, biochemical, and functional analyses has provided evidence that trans and cis binding is mediated by two distinct conformations of Ly49 receptors (Back et al., 2009). Each subunit of the homodimeric Ly 49 receptors is composed of a long stalk region, predicted and in part shown to consist of three short $\alpha$-helical segments, which link the globular NKD to the NK cell membrane. In a back-folded receptor conformation, the two NKDs are associated in an open configuration and this non-covalent dimer is associated with the third $\alpha$-helical segment $\left(\alpha 3_{S}\right)$ of the stalk. There is further evidence that this receptor conformation binds simultaneously two MHC-I molecules expressed on the surface of target cells (in trans) to inhibit NK cell function. Conversely, in an extended receptor conformation, the two NKDs are associated in a closed dimer configuration, which is dissociated from the stalk and in which the orientation of ligand-binding domain is reversed as compared to the back-folded conformation. The extended conformation is thought to bind a single MHC-I molecule expressed on the NK cell itself (in cis; Back et al., 2009; see Figure 1 for illustration).

To validate this model, we addressed whether there is evidence that the unbound and the cis-associated Ly49A receptor adopt distinct conformations on the surface of living cells. To this end, we interrogated the distance between the NKD and the cell membrane using fluorescence resonance energy transfer (FRET). FRET requires close spatial proximity between a donor and an acceptor fluorophore, which is typically a distance of $<100 \AA$ (Kenworthy, 2001). It has been reported that fluorescent membrane dyes can be used to detect conformational changes in membraneanchored proteins by FRET (Chigaev et al., 2003; Jiao et al., 2005; $\mathrm{Xu}$ et al., 2008). Of relevance to the present study, FRET was used to distinguish between the inactive (low affinity) state of $\alpha_{4} \beta_{1}$ integrin, in which the integrin adopts a folded conformation, and the activated (high affinity) state, in which the integrin assumes an extended conformation (Chigaev et al., 2003). In these

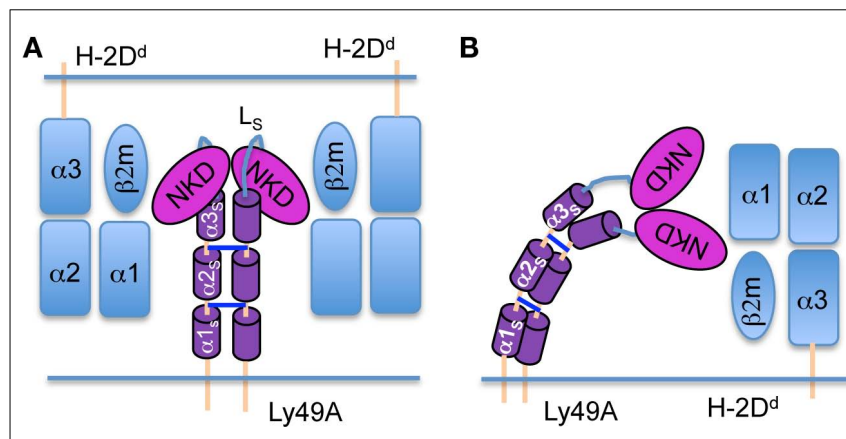

FIGURE 1 | Models of trans and cis interactions of the Ly49A receptor with H-2D ${ }^{\text {d }}$. (A) trans Interaction of the Ly49A NK cell receptor with two $\mathrm{H}-2 \mathrm{D}^{d}$ molecules. The $\mathrm{H}-2 \mathrm{D}^{d}$ molecules are in light blue; the Ly49A NKDs are in red and the $\alpha$-helical segments of the stalk ( $\alpha 1_{s}$ to $\alpha 3_{s}$ ) are in purple. The loop $\left(L_{s}\right)$ connecting $\alpha 3_{s}$ to the NKD is in blue. The NKDs are associated in an open dimer configuration and back-folded onto the stalk to bind two $\mathrm{H}-2 \mathrm{D}^{\mathrm{d}}$ molecules on a target cell. (B) Cis interaction of Ly49A with a single $\mathrm{H}-2 \mathrm{D}^{\mathrm{d}}$ molecule. The Ly49A receptor assumes an extended conformation, which engages a single $\mathrm{H}-2 \mathrm{D}^{\mathrm{d}}$ molecule on the NK cell itself. Consequently, the NKDs are associated in a closed configuration and must dissociate from the stalk. experiments, octadecyl rhodamine B (R18), a lipophilic probe that inserts into the membrane, served as the FRET acceptor. Energy transfer between R18 and $\alpha_{4} \beta_{1}$ integrin labeled with FITC (FRET donor) provided information on the conformational state of the integrin, since FRET efficiencies correlate inversely with the sixth power of the distance of FITC from the plasma membrane. Thus, it was demonstrated that reductions in FRET efficiency were due to vertical extensions of $\alpha_{4} \beta_{1}$ integrin upon activation with divalent cations (Chigaev et al., 2003).

Here we used transfectants expressing wild type or mutant Ly49A receptors in the presence or absence of $\mathrm{H}-2 \mathrm{D}^{\mathrm{d}}$ in cis and determined the FRET efficiency between a fluorescent Fab fragment bound to the Ly49A NKD (FRET donor) and the lipophilic rhodamine dye inserted into the cell membrane (FRET acceptor) to measure changes in the distance between the NKD and the cell membrane. These analyzes provide evidence for distinct conformations of unbound and cis-associated Ly49A receptors on the surface of live cells.

\section{MATERIALS AND METHODS \\ MABS AND FAB FRAGMENTS}

MAb JR9-318 (anti-Ly49A, mIgG1; Roland and Cazenave, 1992) binds the ligand-binding NKD domain of Ly49A (Wang et al., 2002). Fab fragments of mAb JR9 were prepared using the mouse IgG1 Fab preparation kit (Pierce) according to the manufacturer's instructions. Fabs were labeled with Alexa-488 (Invitrogen) using standard conditions. For CD45 FRET, we used biotinylated mAb M1/89 in conjunction with Streptavidin-Alexa-488 (Pierce).

\section{TRANSFECTANTS AND ACID TREATMENT}

C1498 $\left(\mathrm{H}-2^{\mathrm{b}}\right)$ tumor cells stably expressing wild type Ly49A or stalk mutant Ly49A receptors $\left(\Delta \alpha 1_{S}, \Delta \alpha 1-2_{S}\right.$, or $\left.\Delta \mathrm{L}_{\mathrm{S}}\right)$ in the absence and presence of $\mathrm{H}-2 \mathrm{D}^{\mathrm{d}}$ have been described before (Back et al., 2009). The cell surface expression of wild type Ly49A and $\mathrm{H}-2 \mathrm{D}^{\mathrm{d}}$ was comparable to that observed on NK cells (Doucey et al., 2004). The relative expression levels of mutant Ly49A receptors are indicated in Table 1. For the destruction of MHC-I complexes, cells were washed twice in PBS and resuspended in citrate buffer $\left(0.133 \mathrm{M}\right.$ citric acid, $0.066 \mathrm{M} \mathrm{Na}_{2} \mathrm{HPO}_{4}$ at $\left.\mathrm{pH} 3.3\right)$ at $5 \times 10^{6}$ cells $/ \mathrm{ml}$ for $4 \mathrm{~min}$ at room temperature. Exposure to the acidic $\mathrm{pH}$ was terminated by the addition of an excess of $\mathrm{PBS}+5 \%$ FCS. Acid treatment did not reduce the viability of the cells, as judged by trypan blue exclusion or forward and side scatter analysis. Efficient acid stripping was ensured by the loss of the $\beta 2 \mathrm{~m}$ light chain (using mAb Ly-m11) from the cell surface using flow cytometry.

\section{FLOW CYTOMETRY AND FRET}

One million cells were stained with JR9-Fab-Alexa-488 for $20 \mathrm{~min}$ on ice in $100 \mu \mathrm{L}$ of FACS buffer (PBS $+5 \%$ FCS). Half the labeled cells were further stained with R18 (octadecyl rhodamine B, 20$25 \mu \mathrm{M}$ final concentration; Invitrogen) for $4 \mathrm{~min}$ at room temperature. After washing once in cold FACS buffer, the cells were resuspended in cold FACS buffer and analyzed immediately on a FACS can flow cytometer, whereby FL2 overlapping into FL1 (FL1-FL2) was compensated. Data analysis was done using FlowJo software (Tristar). FRET was calculated based on quenching of 
Table 1 | FRET obtained from Ly49A stalk variants.

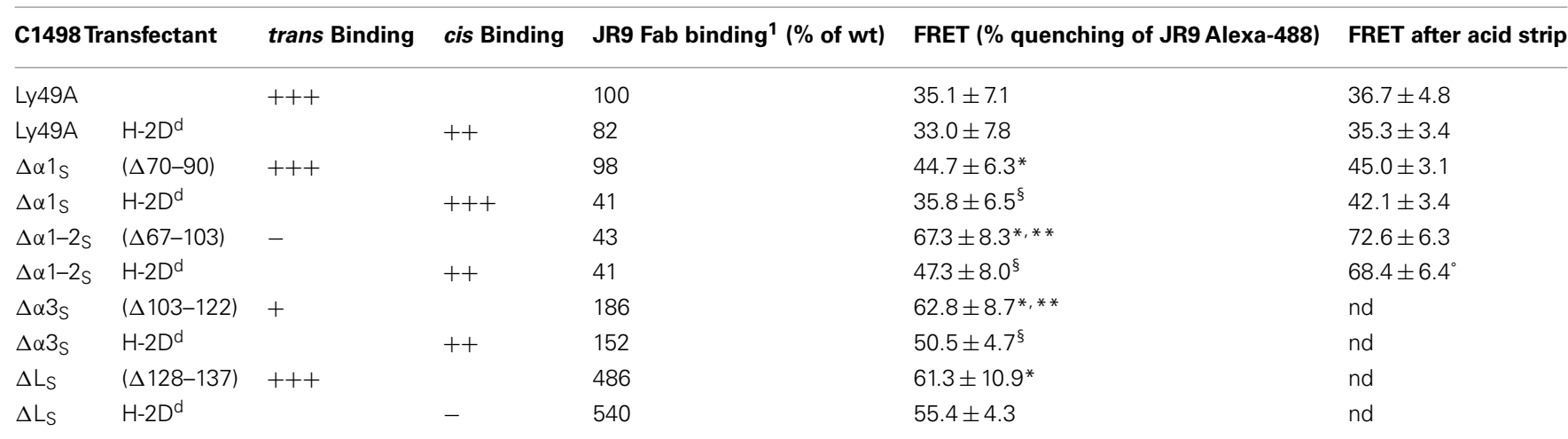

*Significantly different from wild type Ly49A ( $p<0.01)$.

* Significantly different from $\Delta a 1_{s}(p<0.01)$.

s Significantly different from the corresponding variant lacking $\mathrm{H}-2 \mathrm{D}^{d}(p<0.01)$.

'Significantly different from non-stripped ( $p<0.001)$.

Data represent the mean $( \pm S D$ ) of 8-12 independent experiments.

The acid strip data are from 3-4 independent experiments.

nd $=$ not determined.

the Alexa-488 fluorescence by R18 using the following formula: $100 \times$ [mean fluorescence intensity (MFI 488 (no R18) - MFI 488 (with R18)/MFI 488 (no R18)], where MFI 488 refers to the MFI of Alexa-488.

\section{STATISTICAL ANALYSIS}

Statistical analysis was performed using a two-tailed student $t$-test with equal sample variance. Data sets are considered significantly different when $p<0.05$.

\section{RESULTS}

\section{EXPERIMENTAL DESIGN}

Each subunit of the homodimeric Ly49A receptor is composed of an unusually long stalk of 66 amino acids (aa), which links a globular ligand-binding NKD to the NK cell membrane. To interrogate changes in the distance between the NKD and the cell membrane or the orientation of the NKDs relative to the cell membrane, we attached a fluorescent probe to the NKD. To this end, we produced Fab fragments derived from mAb JR9 (Roland and Cazenave, 1992), which binds the NKD of Ly49A (Wang et al., 2002). To serve as a FRET donor, the Fabs were labeled with Alexa-488, which has excitation and emission maxima of $499 \mathrm{~nm}$ and $519 \mathrm{~nm}$, respectively. As a FRET acceptor, we used octadecyl rhodamine B (R18), a plasma membrane dye with excitation and emission maxima at $540 \mathrm{~nm}$ and $565 \mathrm{~nm}$, respectively. The efficiency of FRET was determined based on the quenching of Alexa-488 fluorescence (FL1) by R18 (FL2) at the single cell level using flow cytometry.

\section{SHORTENING OF THE LY49A STALK RESULTS IN INCREASED FRET}

The experimental strategy was validated using transfectants $\left(\mathrm{H}-2^{\mathrm{b}}\right.$ background) expressing full-length Ly49A (66 aa stalk; 67-133) or variants in which predicted $\alpha$-helical segments in the stalk $\left(\alpha_{S}\right)$ had been deleted: $\Delta \alpha 1_{S},(\Delta 70-90 ; 45$ aa stalk) and $\Delta \alpha 1-$ $2_{S},(\Delta 67-103 ; 30$ aa stalk; Figure $2 A)$. The shortening of the stalk decreases the distance between the NKD and the cell membrane and should thus increase FRET between NKD-bound and membrane-associated FRET pairs (Figure 2A). We have previously shown that these stalk deletion mutants bind mAb JR9 and $\mathrm{H}$ $2 \mathrm{D}^{\mathrm{d}}$ multimers (Back et al., 2009), demonstrating that the NKDs are properly folded and competent to bind MHC-I. After labeling cells expressing wild type Ly49A with the two fluorophores, we observed a significant quenching of Alexa-488 fluorescence, indicative of FRET (35.1 $\pm 7.1 \%$ FRET; Figures 2B,C). Comparable FRET was obtained with a second, independently produced batch of JR9 Fab (not shown). FRET was significantly increased when the Ly49A stalk was shortened $\left[\Delta \alpha 1_{S}(44.7 \pm 6.3 \%)\right.$ and $\Delta \alpha 1-2_{S}(67.3 \pm 8.3 \%)$ ] (Figures $2 B, C$ ), as expected by a reduced distance between the NKD and the cell membrane.

Since distinct transfectants vary in cell surface Ly49A levels, we addressed whether FRET was influenced by the intensity of Alexa-488 fluorescence. However, FRET remained essentially constant upon titrating the JR9 Fab (Figure 3) demonstrating that FRET is largely independent of the intensity of JR9 Fab staining and thus independent of Ly49A cell surface levels. Further, FRET obtained with wild type Ly49A was compared to that of CD45, a large cell surface molecule. Significantly less FRET was obtained using CD45 (11.9 $\pm 1.0 \%)$ as compared to full-length Ly49A ( $24.5 \pm 6.0 \%$; data not shown). Residual FRET is expected even with very large cell surface molecules depending on the freedom of the ectodomain to reposition relative to the cell membrane. Indeed, no FRET signal is observed when the Alexa-488 and the R18 labels are on separate cells that are mixed just prior to the analysis (Figure 2B).

\section{THE PRESENCE OF H-2DD IN CIS RESULTS IN REDUCED FRET}

We next determined whether FRET was modified when Ly49A was co-expressed with its cognate MHC-I ligand $\mathrm{H}-2 \mathrm{D}^{\mathrm{d}}$. Importantly, the association of Ly49A with $\mathrm{H}-2 \mathrm{D}^{\mathrm{d}}$ in cis does not prevent the binding of mAb JR9 to Ly49A, although the staining is modestly reduced (50\%). Using full-length Ly49A, there was no difference in FRET between transfectants co-expressing and 


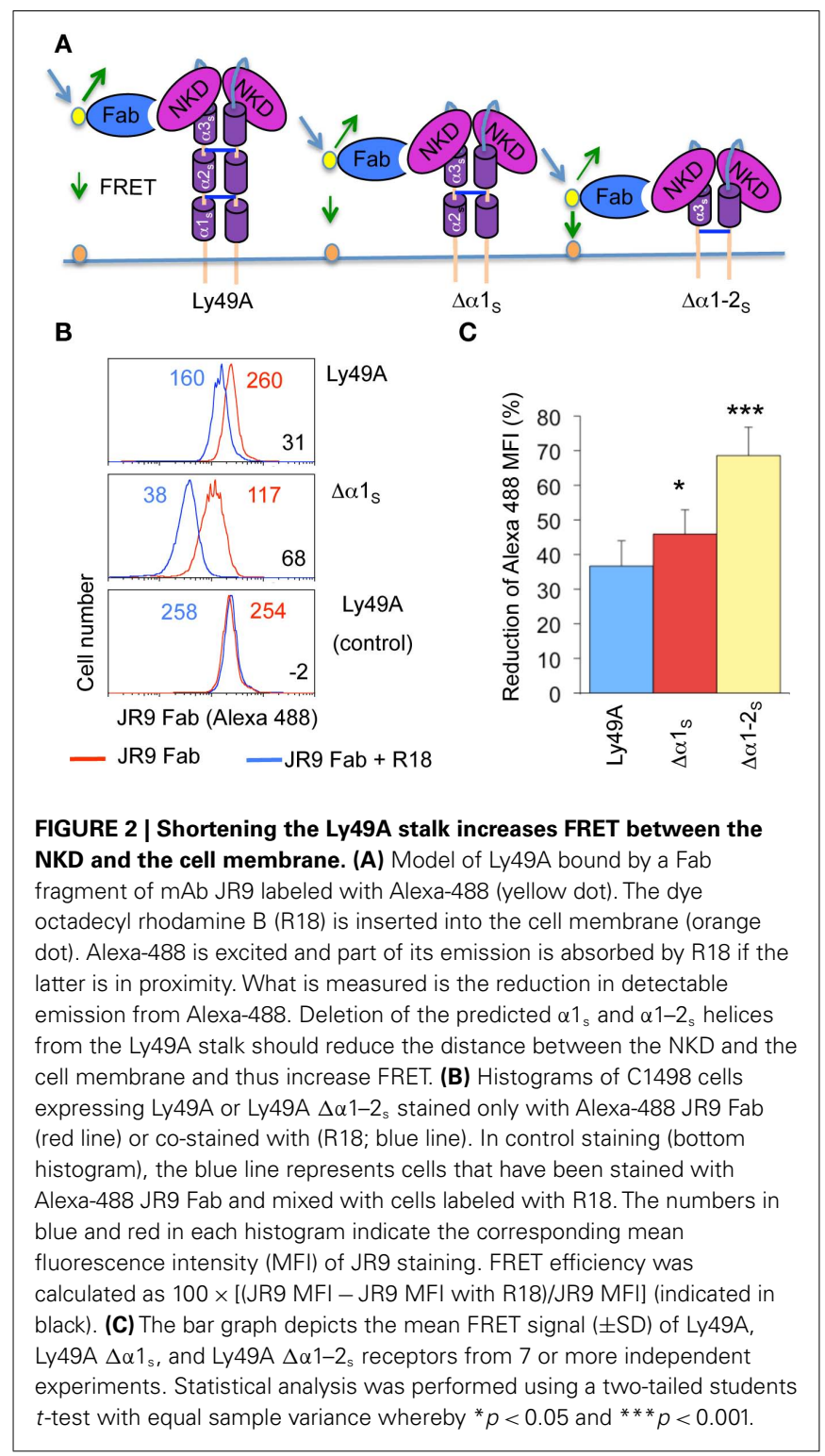

lacking $\mathrm{H}-2 \mathrm{D}^{\mathrm{d}}$ (Figure 4A,B). However, the presence of $\mathrm{H}-2 \mathrm{D}^{\mathrm{d}}$ significantly reduced FRET obtained from Ly49A receptors with shortened stalk regions (i.e., $\Delta \alpha 1_{S}$ and $\Delta \alpha 1-2_{S}$; Figure $4 A, B$ ), suggesting that Ly49A adopts a distinct conformation due to its association with $\mathrm{H}-2 \mathrm{D}^{\mathrm{d}}$ in cis.

cis Association can be disrupted by acid-mediated destruction of MHC-I complexes. Acid treatment of the transfectant expressing wild type Ly49A and H-2D ${ }^{\mathrm{d}}$ did not change FRET while Ly49A $\Delta \alpha 1_{S}$ yielded slightly increased (but not statistically significant) FRET. However, FRET improved significantly upon stripping the Ly49A $\Delta \alpha 1-2 \mathrm{~S} H-2 \mathrm{D}^{\mathrm{d}}$ transfectant (Table 1). Importantly, FRET did not improve when the Ly49A $\Delta \alpha 1-2 \mathrm{~S}$ variant was expressed in the absence of $\mathrm{H}-2 \mathrm{D}^{\mathrm{d}}$ (Table 1). Receptors with a shortened stalk thus provide direct evidence that Ly49A undergoes a significant conformational change due to the association with $\mathrm{H}-2 \mathrm{D}^{\mathrm{d}}$ in cis. The $\mathrm{H}-2 \mathrm{D}^{\mathrm{d}}$-dependent decrease of FRET suggests that the NKDs are relatively distant from the cell membrane when Ly49A
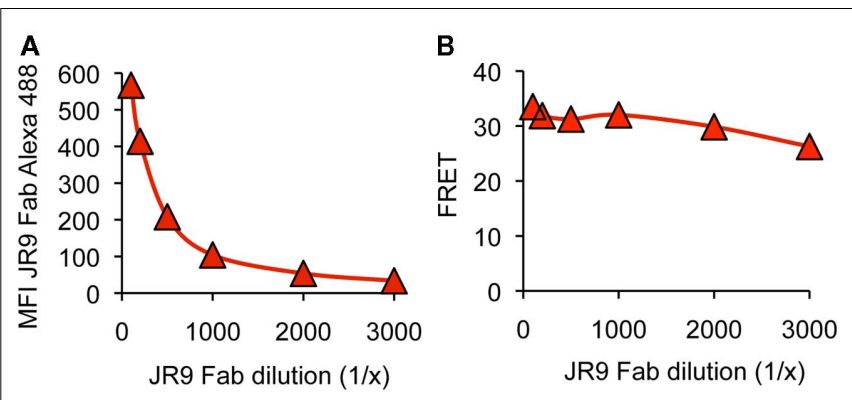

FIGURE 3 | FRET is not modified by variable donor fluorescence. (A) C1498 cells expressing Ly49A were stained with decreasing concentrations of JR9 Fab and the mean fluorescence intensity (MFI) of Alexa-488 fluorescence was recorded. (B) The FRET signal was calculated for each concentration of JR9 Fab. Corresponding data were obtained using two other Ly49A receptors. The data shown are representative of two experiments performed.

is associated with $\mathrm{H}-2 \mathrm{D}^{\mathrm{d}}$ in cis and relatively membrane proximal when the receptor is unbound. This is in very good agreement with the model that the unbound receptor has a back-folded conformation, while cis binding is mediated by an extended receptor.

\section{DELETION OF STALK ELEMENTS REQUIRED FOR LY49A TRANS OR CIS BINDING}

Next we probed the conformation of Ly49A receptors lacking structural elements, which are involved in positioning the NKDs relative to the stalk. The crystal structure of Ly49L revealed that the NKDs make numerous contacts with a $\alpha$ helical segment of the stalk $\left(\alpha 3_{S}\right)$ and that the NKDs are linked to the $\alpha 3_{S}$ using a flexible 12-residue loop ( $\mathrm{L}_{S}$; Back et al., 2009).

The deletion of $\alpha 3_{S}$ impaired trans binding by Ly49A suggesting that a $\Delta \alpha 3_{S}$ receptor cannot adopt a back-folded receptor conformation. Somewhat unexpectedly, the $\Delta \alpha 3_{S}$ receptor yielded significantly increased FRET as compared to the wild type or the $\Delta \alpha 1_{S}$ receptor (Table 1). Since the deletions of $\Delta \alpha 1_{S}$ and $\Delta \alpha 3_{S}$ are of similar size the increased FRET cannot simply be attributed to the shortening of the stalk. While the possible reason for the increase will be discussed below, the data show that the deletion of an element putatively involved in orienting the NKDs relative to the stalk significantly alters the conformation of an unbound receptor. The presence of $\mathrm{H}-2 \mathrm{D}^{\mathrm{d}}$ significantly reduced FRET obtained with the $\Delta \alpha 3_{S}$ receptor, indicating a further conformational change due to the presence of MHC-I ligand. This agrees with our previous findings that the deletion of $\alpha 3_{S}$ is compatible with cis binding by Ly49A implying that the $\Delta \alpha 3_{S}$ receptor can adopt an extended receptor conformation.

The deletion of the $\mathrm{L}_{\mathrm{S}}$ loop, which links the NKDs to the $\alpha 3_{\mathrm{S}}$ element, was compatible with trans binding but incompatible with cis binding (Back et al., 2009). This led us to propose that the deletion of $\mathrm{L}_{S}$ locked Ly49A in a back-folded conformation, unable to adopt an extended receptor conformation.

Again somewhat unexpectedly, we observed significantly increased FRET for the $\Delta \mathrm{L}_{\mathrm{S}}$ as compared to the wild type Ly49A receptor (Table 1). Cell surface expression of this variant was approximately five times higher than that of wild type Ly49A. 


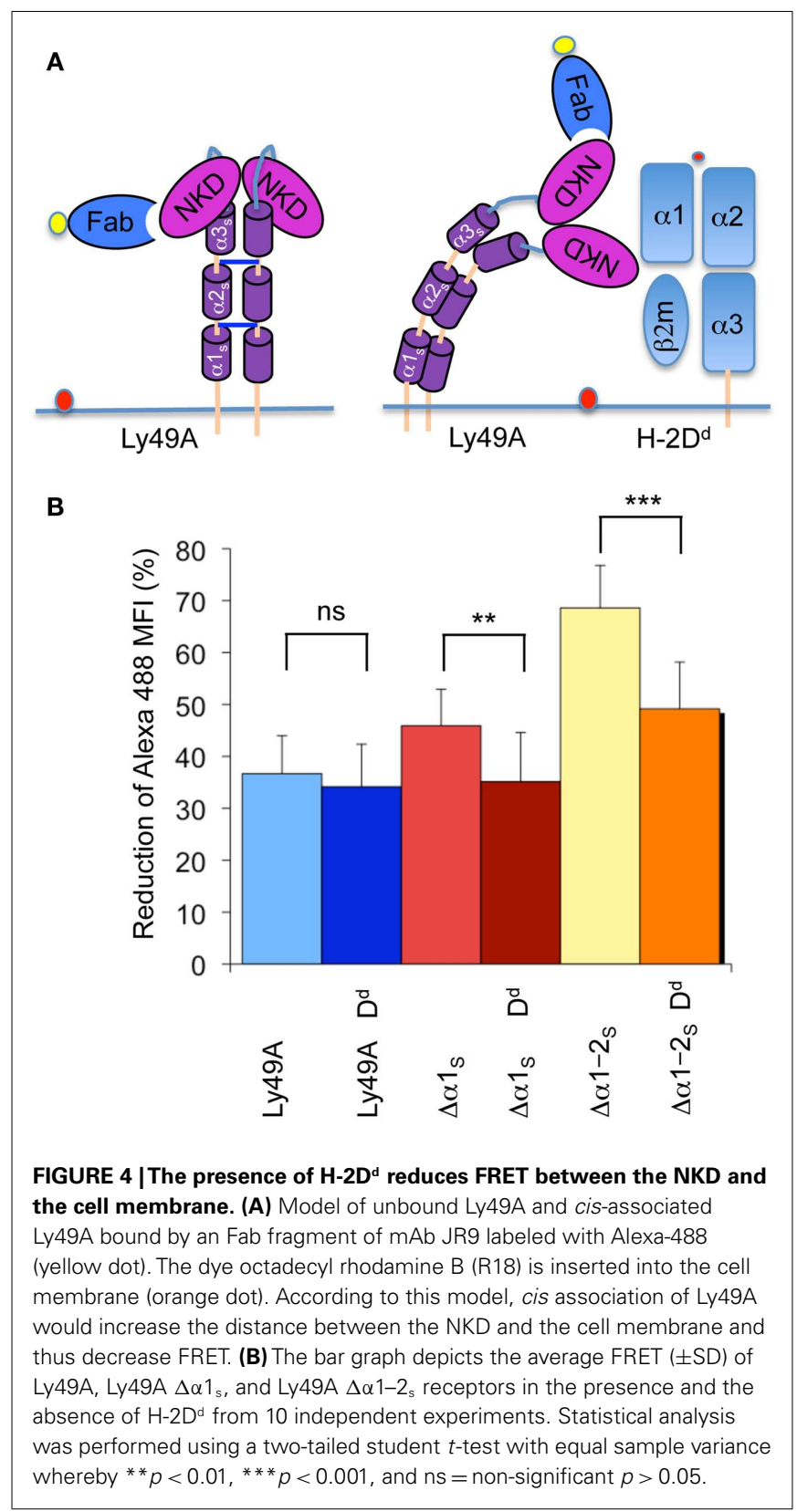

However, corresponding results were obtained when the JR9 Fab was diluted to yield a fluorescence intensity similar to that obtained with wild type Ly49A (not shown). While the putative reason for the increase is discussed below, the data show that the deletion of a second stalk element putatively involved in orienting the NKDs relative to the stalk significantly alters the conformation of the unbound receptor. Finally, the presence of $\mathrm{H}-2 \mathrm{D}^{\mathrm{d}}$ did not alter FRET by the $\Delta \mathrm{L}_{S}$ receptor. This agrees with our previous data showing that the $\Delta \mathrm{L}_{\mathrm{S}}$ receptor cannot bind $\mathrm{H}-2 \mathrm{D}^{\mathrm{d}}$ in cis (Back et al., 2009).

\section{DISCUSSION}

The structural basis for the ability of certain Ly49 receptors to bind MHC-I molecules both in trans and in cis, using a single binding site, is not well understood. We have proposed that the two types of interactions are mediated by distinct receptor conformations, in which the orientation of NKDs is reversed relative to the NK cell membrane (Back et al., 2009). Here we show, that the presence of $\mathrm{H}-2 \mathrm{D}^{\mathrm{d}}$ modifies FRET between fluorescent probes attached to the ligand-binding NKD of Ly49A and inserted into the cell membrane. These data strongly suggest that the Ly49A receptor adopts a distinct conformation upon association with $\mathrm{H}-2 \mathrm{D}^{\mathrm{d}}$ in cis. The decrease in FRET suggests that the NKDs are positioned more distant to the cell membrane when Ly49A is associated with $\mathrm{H}-2 \mathrm{D}^{\mathrm{d}}$ in cis as compared to the unbound receptor. This supports the model that the unbound receptor has a back-folded conformation, while cis binding is mediated by an extended receptor. Of note, changes in FRET based on the presence/absence of $H-2 D^{d}$ are only evident when receptors with shortened stalk regions are analyzed. The Ly49A receptor with a full-size stalk yields comparable FRET in the presence and absence of $\mathrm{H}-2 \mathrm{D}^{\mathrm{d}}$. This is not necessarily inconsistent with the idea of a structural difference in cis-bound versus unbound Ly49A. Rather, the NKDs of a full-size receptor may simply be at a comparable distance from the cell membrane in the back-folded and in the extended receptor conformation. Indeed, due to the length of the stalk (and as depicted in Figure 1), an extended receptor likely needs to "bend over" in order to bind $\mathrm{H}-2 \mathrm{D}^{\mathrm{d}}$ in cis. From this model, it is easy to envisage how extended Ly49 receptors with shortened stalks can bind cis ligand whereby the NKDs remain at a relatively constant distance from the cell membrane (i.e., FRET remains low). In contrast, the NKDs of unbound and back-folded receptors are brought into proximity to the cell membrane when the stalk is shortened (i.e., FRET is increased). Thus, these data strongly support the view that the unbound and the cis-bound Ly49A receptors have extended and back-folded conformations, respectively.

We have further probed the structure of Ly49A receptors lacking elements that are needed to position the NKDs relative to the stalk. Such elements were identified based on the crystal structure of Ly49L, in which the NKDs are associated with $\alpha 3_{\mathrm{S}}$ segment of the stalk. Indeed the deletion of $\Delta \alpha 3_{S}$ was incompatible with trans binding (Back et al., 2009) likely based on the failure of this receptor to stably adopt a back-folded conformation. This deletion variant yielded surprisingly efficient FRET. Indeed, the signal significantly exceeded that obtained upon deleting the $\alpha 1_{S}$ element of the stalk, which is of similar length, showing that $\alpha 3_{S}$ contributes not only to the length of the stalk. One possible explanation for the increased FRET is that the deletion of $\alpha 3_{S}$ abrogates the stable association of the NKDs with the stalk. Based on the flexible $\mathrm{L}_{S}$ loop the NKDs have now a large degree of freedom to move and are thus more likely in proximity to the membrane as compared to a stably back-folded wild type receptor. In agreement with this idea, FRET obtained from the $\Delta \alpha 3_{S}$ receptor is reduced by the presence of $\mathrm{H}-2 \mathrm{D}^{\mathrm{d}}$. The presence of the cognate ligand in cis may stabilize the receptor and position the NKDs in a set distance form the cell membrane.

Finally, the Ly49L crystal structure identified a flexible 12residue $\mathrm{L}_{S}$ loop, which connects the $\alpha 3_{S}$ of the stalk to the NKD. The deletion of $\Delta \alpha 3_{S}$ was compatible with trans but incompatible with cis binding (Back et al., 2009), suggesting that this receptor is locked in a back-folded conformation. The $\Delta \mathrm{L}_{\mathrm{S}}$ receptor 
variant yielded significantly increased FRET as compared to the wild type receptor, even though the vertical distance between the NKD and the cell membrane was not thought to be very different. One explanation may be that the population of wild type Ly49A receptors on a single cell includes both back-folded and extended receptors. If the $\mathrm{L}_{\mathrm{S}}$-deletion receptor existed only in a back-folded conformation this could result in increased FRET. Alternatively, the deletion of $\mathrm{L}_{S}$ may result in a rotation of the NKDs, which reorients the Fab toward the cell membrane. Whatever the precise basis, it is noteworthy that the conformational alteration correlates with the inability of the $\Delta \mathrm{L}_{\mathrm{S}}$ receptor to inhibit NK cell function, even though this receptor can bind MHC-I expressed on other cells (Back et al., 2009).

We have previously reported that cis complexes are remarkably stable (Back et al., 2007). Indeed, there is no evidence that trans ligand can compete with cis ligand for Ly49A binding. The basis for the apparent stability of cis complexes can be rationalized based on our structural analyses. Multiple sequential steps seem to be needed to convert a cis-bound, extended into an unbound back-folded receptor. First, the extended receptor needs to dissociate from cis-bound MHC-I. Although, the receptor will dissociate relatively rapidly from the single MHC-I molecule bound in cis, a high local concentration of MHC-I molecules in cis will likely favor rapid re-association. Once detached from MHC-I, the NKDs, which are non-covalently associated in a closed dimer configuration, will need to dissociate. They would then have to back-fold onto the stalk and re-associate in a distinct, open, dimer conformation. The requirement for this sequence of events may explain why there is no evidence that an extended receptor can convert into a back-folded receptor conformation under physiological conditions. Notwithstanding, acid stripping may be able to promote this conversion. Acid stripping of live cells disrupts trimolecular MHC-I complexes acid treatment and "produces" receptors that

\section{REFERENCES}

Anfossi, N., Andre, P., Guia, S., Falk, C. S., Roetynck, S., Stewart, C. A., Breso, V., Frassati, C., Reviron, D., Middleton, D., Romagné, F., Ugolini, S., and Vivier, E. (2006). Human NK cell education by inhibitory receptors for MHC class I. Immunity 25, 331-342.

Back, J., Chalifour, A., Scarpellino, L., and Held, W. (2007). Stable masking by H-2Dd cis ligand limits Ly49A relocalization to the site of $\mathrm{NK}$ cell/target cell contact. Proc. Natl. Acad. Sci. U.S.A. 104, 3978-3983.

Back, J., Malchiodi, E. L., Cho, S., Scarpellino, L., Schneider, P., Kerzic, M. C., Mariuzza, R. A., and Held, W. (2009). Distinct conformations of Ly49 natural killer cell receptors mediate MHC class I recognition in trans and cis. Immunity 31, 598-608.

Chalifour, A., Scarpellino, L., Back, J., Brodin, P., Devèvre, E., Gros, F., Lévy, F., Leclercq, G., Höglund, P., Beermann, F., and Held, W. (2009). A role for cis interaction between the inhibitory Ly49A receptor and
MHC class I for NK cell education. Immunity 30, 337-347.

Chigaev, A., Buranda, T., Dwyer, D. C., Prossnitz, E. R., and Sklar, L. A. (2003). FRET detection of cellular alpha4-integrin conformational activation. Biophys. J. 85, 3951-3962.

Doucey, M. A., Scarpellino, L., Zimmer, J., Guillaume, P., Luescher, I. F., Bron, C., and Held, W. (2004). Cis-association of Ly49A with MHC class I restricts natural killer cell inhibition. Nat. Immunol. 5, 328-336.

Fernandez, N. C., Treiner, E., Vance, R. E., Jamieson, A. M., Lemieux, S., and Raulet, D. H. (2005). A subset of natural killer cells achieve self-tolerance without expressing inhibitory receptors specific for self MHC molecules. Blood 105, 4416-4423.

Held, W., and Mariuzza, R. A. (2008). Cis interactions of immunoreceptors with MHC and non-MHC ligands. Nat. Rev. Immunol. 8, 269-278.

Jiao, X., Zhang, N., Xu, X., Oppenheim, J. J., and Jin, T. (2005). Ligandinduced partitioning of human

can bind MHC-I in trans (Back et al., 2007). Acid treatment may thus force the dissociation of the two NKDs in the extended receptor confirmation, which may then re-associate, at least in part, in the back-folded conformation. The data presented herein do support this idea. Acid stripping of a transfectant expressing a Ly49A variant with a short stalk and $\mathrm{H}-2 \mathrm{D}^{\mathrm{d}}$ results in a significant increase in FRET, consistent with back-folding of previously extended receptors.

Consequently cis binding of Ly49A stably reduces the number of receptors that can productively interact with $\mathrm{D}^{\mathrm{d}}$ expressed on potential target cells (Back et al., 2007). There is no evidence that cis binding of Ly49A results in inhibitory signaling (Doucey et al., 2004). Hence, cis binding lowers the threshold at which NK cell activation exceeds inhibition. This renders Ly49A NK cells more sensitive to react to diseased host cells. Further data suggest that cis interaction of Ly49A with MHC-I plays an essential role in NK cell education (Chalifour et al., 2009; Jonsson et al., 2010). Here, the sequestration of unbound Ly49 receptors contributes to an improved function of NK cell activation receptors (Chalifour et al., 2009). It remains possible, however, that cis complexes are more directly implicated in NK cell education. Irrespectively, cis binding of Ly49 receptors stably adapts the function of NK cells to the inherited MHC-I haplotype.

In conclusion, we have developed a novel FRET based approach to study conformational changes in cell surface receptors, which provides evidence based on the analysis of live cells that $c i s$ and trans binding of MHC-I is mediated by distinct conformations of Ly49 receptors.

\section{ACKNOWLEDGMENTS}

This work was supported in part by grants from the Swiss National Science Foundation (to Werner Held) and the National Institutes of Health (to Roy A. Mariuzza).

CXCR1 chemokine receptors with lipid raft microenvironments facilitates G-protein-dependent signaling. Mol. Cell. Biol. 25, 5752-5762.

Jonsson, A. H., Yang, L., Kim, S., Taffner, S. M., and Yokoyama, W. M. (2010). Effects of MHC class I alleles on licensing of Ly49A+ NK cells. J. Immunol. 184, 3424-3432.

Karlhofer, F. M., Hunziker, R., Reichlin, A., Margulies, D. H., and Yokoyama, W. M. (1994). Host MHC class I molecules modulate in vivo expression of a NK cell receptor. J. Immunol. 153, 2407-2416.

Kenworthy, A. K. (2001). Imaging protein-protein interactions using fluorescence resonance energy transfer microscopy. Methods 24, 289-296.

Kim, S., Poursine-Laurent, J., Truscott, S. M., Lybarger, L., Song, Y. J., Yang, L., French, A. R., Sunwoo, J. B., Lemieux, S., Hansen, T. H., and Yokoyama, W. M. (2005). Licensing of natural killer cells by host major histocompatibility complex class I molecules. Nature 436, 709-713.

Lanier, L. L. (2005). NK cell recognition. Annu. Rev. Immunol. 23, 225-274.

Masuda, A., Nakamura, A., Maeda, T., Sakamoto, Y., and Takai, T. (2007). Cis binding between inhibitory receptors and MHC class I can regulate mast cell activation. J. Exp. Med. 204, 907-920.

Roland, J., and Cazenave, P. A. (1992). Ly-49 antigen defines an alpha beta TCR population in i-IEL with an extrathymic maturation. Int. Immunol. 4, 699-706.

Scarpellino, L., Oeschger, F., Guillaume, P., Coudert, J. D., Levy, F., Leclercq, G., and Held, W. (2007). Interactions of Ly49 family receptors with MHC class I ligands in trans and cis. $J$. Immunol. 178, 1277-1284.

Sola, C., Andre, P., Lemmers, C., Fuseri, N., Bonnafous, C., Blery, M., Wagtmann, N. R., Romagne, F., Vivier, E., and Ugolini, S. (2009). Genetic and antibody-mediated reprogramming of natural killer cell missing-self recognition in vivo. 
Proc. Natl. Acad. Sci. U.S.A. 106, 12879-12884.

Wang, J., Whitman, M. C., Natarajan, K., Tormo, J., Mariuzza, R. A., and Margulies, D. H. (2002). Binding of the natural killer cell inhibitory receptor Ly49A to its major histocompatibility complex class I ligand. J. Biol. Chem. 277, 1433-1442.

Xu, C., Gagnon, E., Call, M. E., Schnell, J. R., Schwieters, C. D.,
Carman, C. V., Chou, J. J., and Wucherpfennig, K. W. (2008). Regulation of $\mathrm{T}$ cell receptor activation by dynamic membrane binding of the CD3epsilon cytoplasmic tyrosine-based motif. Cell 135, 702-713.

Conflict of Interest Statement: The authors declare that the research was conducted in the absence of any commercial or financial relationships that could be construed as a potential conflict of interest.

Received: 10 July 2011; accepted: 21 September 2011; published online: 11 October 2011.

Citation: Back J, Angelov GS, Mariuzza $R A$ and Held W (2011) The interaction with $H-2 D^{d}$ in cis is associated with a conformational change in the Ly49A NK cell receptor. Front. Immun. 2:55. doi: 10.3389/fimmu.2011.00055
This article was submitted to Frontiers in NK Cell Biology, a specialty of Frontiers in Immunology.

Copyright (๑ 2011 Back, Angelov, Mariuzza and Held. This is an open-access article subject to a non-exclusive license between the authors and Frontiers Media $S A$, which permits use, distribution and reproduction in other forums, provided the original authors and source are credited and other Frontiers conditions are complied with. 